\title{
Serum galectin-I in patients with multiple myeloma: associations with survival, angiogenesis, and biomarkers of macrophage activation
}

This article was published in the following Dove Press journal:

OncoTargets and Therapy

4 April 2017

Number of times this article has been viewed

Morten Nørgaard

Andersen ${ }^{1-3, *}$

Maja Ludvigsen ${ }^{1,3, *}$

Niels Abildgaard ${ }^{4}$

Irma Petruskevicius ${ }^{3}$

Rikke Hjortebjerg ${ }^{5}$

Mette Bjerre ${ }^{5}$

Bent Honoré

Holger J Møller ${ }^{2}$

Niels F Andersen ${ }^{3}$

'Department of Biomedicine, Faculty of Health, Aarhus University, ${ }^{2}$ Department of Clinical Biochemistry, ${ }^{3}$ Department of Hematology, Aarhus University Hospital, Aarhus, ${ }^{4}$ Department of Hematology, Odense University Hospital, Odense, ${ }^{5}$ Medical Research Laboratory, Department of Clinical Medicine, Faculty of Health, Aarhus University, Aarhus, Denmark

*These authors contributed equally to this work
Correspondence: Morten Nørgaard Andersen

Department of Biomedicine, Aarhus University, Wilhelm Meyers Allé 4, Aarhus C 8000, Denmark Email morten@immunology.au.dk
Abstract: Galectin-1 (Gal-1) is known to regulate cell signaling within the immune system and may be a target for new anticancer immune therapy. In patients with chronic lymphocytic leukemia (CLL) and classical Hodgkin lymphoma (cHL), high levels of Gal-1 within the tumor microenvironment were associated with worse disease state or poor outcome. Gal-1 can be secreted from cells by an unknown mechanism, and levels in blood samples were associated with high tumor burden and worse disease state in cHL and CLL patients. However, serum levels of Gal-1 have never been investigated in patients with multiple myeloma (MM). We measured serum Gal-1 levels in samples from patients with treatment demanding MM at the time of diagnosis $(n=102)$ and after treatment $(n=24)$ and examined associations of serum Gal-1 with clinicopathological information obtained from patient medical records, as well as data on bone marrow angiogenesis and the macrophage activation biomarkers soluble CD163 (sCD163) and soluble mannose receptor. Serum Gal-1 levels were not elevated in patients with MM at diagnosis compared with healthy donors (median values 8.48 vs $11.93 \mathrm{ng} / \mathrm{mL}, P=0.05$ ), which is in contrast to results in cHL and CLL. Furthermore, Gal-1 levels did not show association with bone marrow angiogenesis, clinicopathological parameters, overall survival, or response to treatment. There was a statically significant association between Gal-1 and sCD163 levels ( $R=0.24, P=0.02$ ), but not with soluble mannose receptor $(P=0.92)$. In conclusion, our results indicate that Gal-1 is not an important serum biomarker in MM, which is in contrast to data from patients with cHL and CLL. However, the association with SCD163 is in line with previous data showing that Gal-1 may be involved in alternative (M2-like) activation of macrophages.

Keywords: galectin-1, multiple myeloma, macrophage, soluble CD163, soluble mannose receptor, angiogenesis

\section{Introduction}

Galectins comprise a 15-member family of lectins (carbohydrate-binding proteins), of which some are widely distributed in various tissues and others are more confined. ${ }^{1,2}$ Galectins exert diverse functions by binding to carbohydrate moieties on, for example, extracellular proteins, but they also function intracellularly where they are involved in cell signaling processes. ${ }^{1}$ Furthermore, galectins have been linked with the regulation of hematopoiesis and modulation of the immune system in both acute and chronic inflammation. ${ }^{1,3}$

Galectin-1 (Gal-1) is involved in regulating homeostasis and differentiation of both $\mathrm{T}$ and $\mathrm{B}$ cells, can skew an immune reaction in a $\mathrm{T}_{\mathrm{H}}$ 2-like (anti-inflammatory) direction, and may sway monocyte/macrophage polarization toward an alternatively activated (M2-like) phenotype. ${ }^{4,5}$ Importantly, studies have indicated that inhibition 
of Gal-1 signaling may affect immune activation, and thus could be a strategy for new anticancer therapy.,

In a study on classical Hodgkin lymphoma (cHL) patients, Gal-1 was expressed by Hodgkin and Reed-Sternberg cells as well as macrophages and endothelial cells. Interestingly, high expression of Gal-1 within the tumor microenvironment was associated with poor survival. ${ }^{8}$

Gal-1 can be secreted from cells by an unknown mechanism $^{1}$ and can be measured in blood samples..$^{9,10}$ In patients with chronic lymphocytic leukemia (CLL), both bone marrow and plasma levels of Gal-1 were increased in patients with progressive compared with stable disease, and CLL-supporting myeloid cells were found to be the major producers of Gal-1. ${ }^{11}$ In cHL patients, serum Gal-1 levels were increased compared with healthy controls and associated with clinical features of high tumor burden. ${ }^{9}$

Multiple myeloma (MM) is the second most common hematological malignancy, in which malignant plasma cells (PCs) proliferate within the bone marrow, leading to anemia, bone destruction, and renal failure. ${ }^{12}$ The bone marrow microenvironment is known to be of major importance in the disease. Recently, macrophages have been reported as important supportive cells in MM, at least in part because of the promotion of $\mathrm{MM}$ cell growth and resistance to therapy, ${ }^{13}$ and infiltration by $\mathrm{CD} 163^{+}$macrophages in the bone marrow is associated with poor outcome in MM. ${ }^{14,15}$ Within the few reports addressing the role of galectins in MM, one study indicated that Gal-1 increased proliferation and survival of CD45RA ${ }^{\text {neg }} \mathrm{MM}$ cells. ${ }^{16} \mathrm{~A}$ recent study reported increased Gal-1 mRNA expression in CD138+ bone marrow cells of MM patients, and that Gal-1 may be important in MM-induced angiogenesis. ${ }^{17}$ Increased bone marrow angiogenesis is a characteristic feature of MM, where high level of angiogenesis is associated with poor outcome, ${ }^{18}$ and interestingly, Gal-1 may be involved in the regulation of angiogenesis in various human cancers. ${ }^{19}$

However, levels of serum Gal-1 have never been reported for patients with MM. Therefore, the aim of this study was to determine Gal-1 levels in patients with newly diagnosed $\mathrm{MM}$ and investigate associations with clinicopathological parameters, bone marrow angiogenesis, markers of macrophage activation, and patient outcome.

\section{Patients and methods}

\section{Patients}

The cohort used in this study consists of 102 patients with newly diagnosed, treatment demanding MM, included at the Department of Hematology, Aarhus University Hospital,
Aarhus, Denmark, from 1991 to 2004. With data from the same cohort of patients, we have previously published results on the macrophage-derived biomarkers soluble CD163 and soluble CD206/mannose receptor (sCD163 and sMR). ${ }^{20,21}$ Basic characteristics of the included patients have been thoroughly described previously. ${ }^{20}$ Briefly, patients were treated with either conventional peroral chemotherapy (e.g., melphalan) and prednisolone (MP, 60\%) or high-dose melphalan with autologous stem cells support (high dose therapy [HDT], 40\%). We obtained a serum sample from the time of diagnosis and measured Gal- 1 concentration. Of the included patients, 54\% were male, median age at diagnosis was 60 years (range 39-84 years), and the heavy chain class subtypes were IgG: 58\%, IgA: 25\%, and light chain only: $17 \%$. Furthermore, for 24 patients, we were able to measure serum Gal-1 in a sample taken 3 months after HDT. Data from a previously published study in which we have measured serum Gal-1 in 30 healthy donors were included as a control group: 53\% male, median age 53 years (range $45-66$ years)..$^{10}$ All samples were stored at $-80^{\circ} \mathrm{C}$.

Information about basic characteristics, pathology, and biochemistry results was obtained from patient medical records. Response to treatment was categorized as 1) complete response (CR) or 2) less than CR. CR was defined as negative serum and urine $\mathrm{M}$-protein by electrophoresis and $<5 \%$ PCs in the bone marrow. Data on bone marrow angiogenesis, measured as $\mathrm{CD}^{+} 4^{+}$micro-vessel density (MVD), were obtained during a previous study and are described in detail in the study by Andersen et al. ${ }^{18}$ The study was approved by the central Denmark region committee on health research ethics (M-20100171). The requirement for study-specific informed consent was waived due to the retrospective nature of the study and the long follow-up time since inclusion.

\section{Measurement of serum Gal-I}

Serum Gal-1 levels were measured using a time-resolved immunofluorometric assay as described previously. ${ }^{10}$ Microtiter wells were coated with capture antibody (AF1152, R\&D Systems) at $2 \mu \mathrm{g} / \mathrm{mL}$ in phosphate-buffered saline (PBS)/pH 8 for $2 \mathrm{~h}$. Plates were washed and blocked for $1 \mathrm{~h}$ (PBS, pH 7.4, 1\% [v/v] Tween20). The wells were washed and standards or diluted serum (1:4) were added (dilution in assay buffer: PBS, pH 7.4, $25 \mu \mathrm{M}$ ethylenediaminetetraacetic acid, $0.05 \%[\mathrm{v} / \mathrm{v}]$ human serum albumin, $1 \%[\mathrm{v} / \mathrm{v}]$ Tween 20 , $0.9 \%[\mathrm{w} / \mathrm{v}] \mathrm{NaCl}$ ) and incubated for $2 \mathrm{~h}$ at room temperature. Following incubation with biotin-conjugated detection antibody (BAF1152, R\&D Systems) at $0.1 \mu \mathrm{g} / \mathrm{mL}$ in assay buffer supplemented with europium-labeled streptavidin 
(PerkinElmer) diluted 1:1,000, DELFIA enhancement solution (PerkinElmer) was added and plates were read in a timeresolved fluorometer (Victor3, PerkinElmer). A standard curve of full-length recombinant Gal-1 (1152-GA, R\&D Systems) at concentrations of $100-0.78 \mathrm{ng} / \mathrm{mL}$ was fitted using a 4-parameter nonlinear regression curve for each plate. All samples were run in duplicates, with mean $\% \mathrm{CV}$ of 2.62 in the included samples. Two samples were excluded from further analysis due to $\% \mathrm{CV}>10 \%$. Four donor samples were run in duplicate $(\mathrm{CV}<3.5 \%)$ on every plate, yielding a mean inter-plate CV of $15 \%$ (range $7 \%-21 \%$ ). Previous measurements showed stability of Gal-1 over a year of repeated freeze/thaw cycles. Furthermore, when correlating the measured Gal-1 concentration with a storage time at $-80^{\circ} \mathrm{C}$, we found no indication of a time-dependent change in Gal-1 concentration (data not shown). In this study, three and five samples from patients and healthy donors, respectively, showed a concentration above the range of the standard curve (400 ng/mL). These samples were included in the statistical analyses with a concentration of $400 \mathrm{ng} / \mathrm{mL}$.

\section{Statistical analyses}

Statistical analyses were done using Stata version 11 for Mac (StataCorp LP, TX, USA). Figures were made using Prism 5 for Mac (GraphPad software Inc., CA, USA). Differences between groups were tested using paired or unpaired $t$-tests and one-way analysis of variance, when appropriate. Gaussian distribution of data was assessed by Q-Q plots before analysis, and non-normally distributed data were log-transformed before analysis. For investigation of linear associations between parameters, we used Pearson's correlation analyses. Survival analyses were performed using Cox proportional hazards regression. All Gal-1 concentrations are reported as median values with interquartile range in parentheses. $P$-values of $<0.05$ were considered statistically significant.

\section{Results}

\section{Serum Gal-I at the time of diagnosis and after HDT}

Data on the measurements of Gal-1 concentrations in serum samples from 102 patients with newly diagnosed MM and 30 healthy donors are shown in Figure 1. The Gal-1 levels displayed relatively large variation for both patients and controls, with median values: MM, $8.48 \mathrm{ng} / \mathrm{mL}$ (5.75-14.78) and controls, $11.93 \mathrm{ng} / \mathrm{mL}$ (6.23-80.91), which was a borderline statistically significant difference $(P=0.05)$. In Figure 2, Gal-1 levels are shown in relation to international staging system

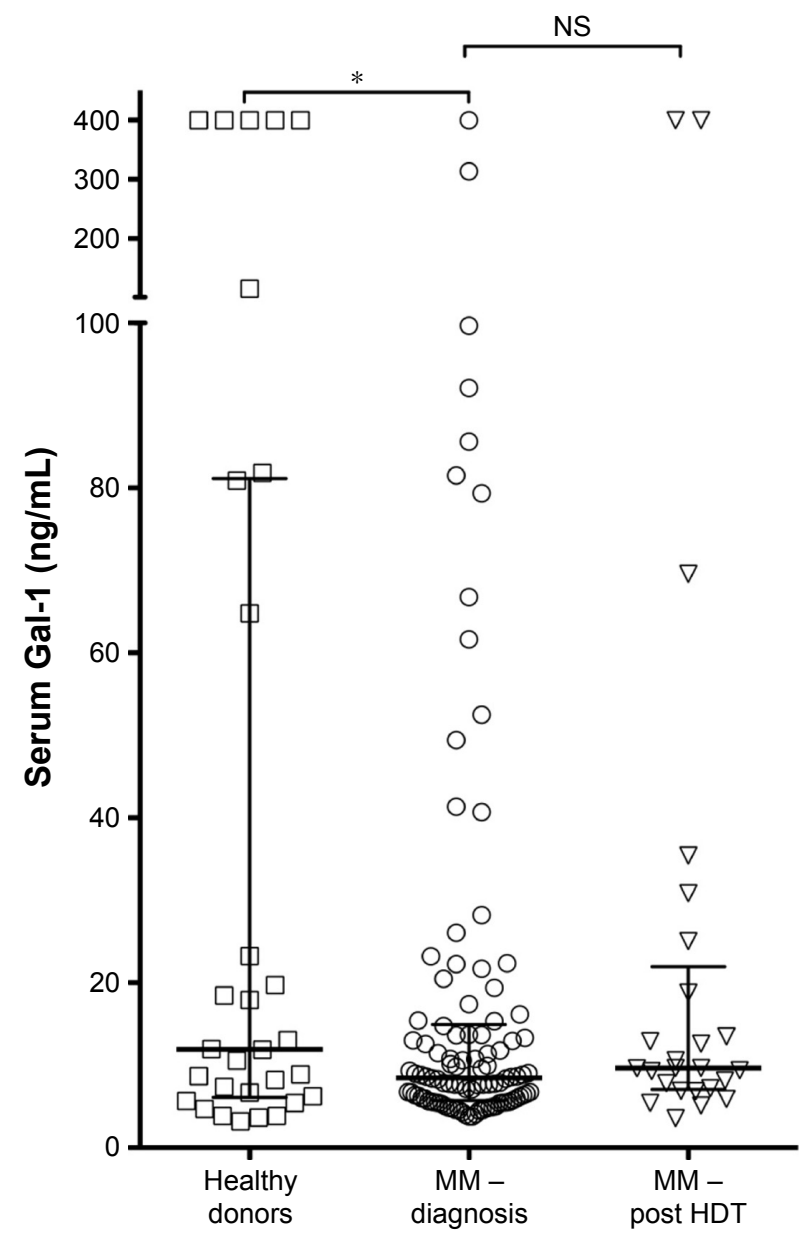

Figure I Serum Gal-I in MM patients pre- and posttreatment.

Notes: Serum Gal-I levels in healthy donors $(n=30)$, patients with newly diagnosed MM ( $n=102)$, and for MM patients 3 months after HDT $(n=24)$. Bars show median and interquartile range. $* P=0.05$.

Abbreviations: Gal-I, galectin-I; HDT, high dose treatment; MM, multiple myeloma; NS, not significant.

stage, ${ }^{22}$ showing no clear association between Gal-1 levels and international staging system stage at the time of diagnosis $(P=0.10)$. Using correlation analyses, we examined associations of Gal-1 with various clinicopathological parameters, but found no statistically significant associations: gender $(P=0.82)$, age at diagnosis $(P=0.18)$, creatinine $(P=0.12)$, albumin $(P=0.31)$, C-reactive protein $(P=0.57)$, beta- 2 microglobulin $(P=0.93)$, and bone marrow $\mathrm{PC}$ infiltration $(P=0.16)$.

In the 24 patients for whom we obtained serial samples, we found no statistically significant difference between Gal-1 levels at diagnosis and 3 months after (Figure 1, $P=0.13$ ).

\section{Association of serum Gal-I levels to treatment response and outcome}

For the 41 patients treated with HDT, we had access to data on the response to treatment. In these patients, there was no association with treatment response for either Gal-1 levels 


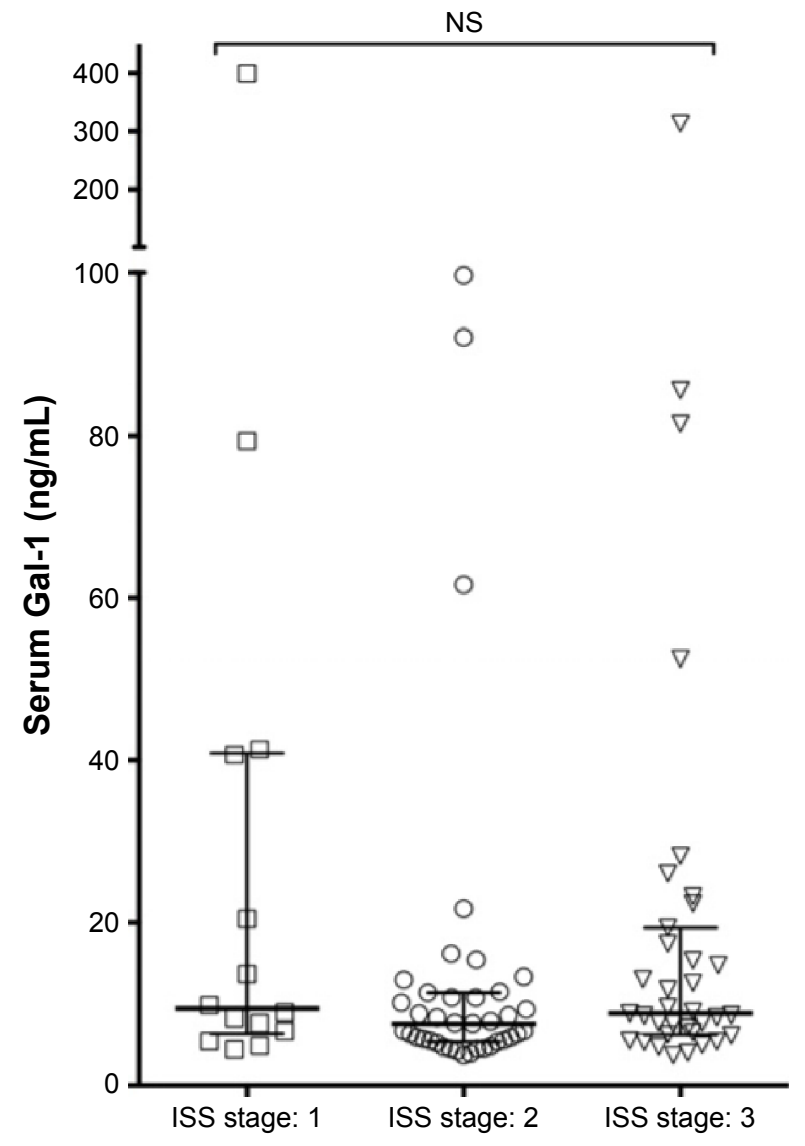

Figure 2 Serum Gal-I in multiple myeloma patients according to ISS stage. Notes: Serum Gal-I levels at time of diagnosis in patients with multiple myeloma, stratified by ISS stage. Bars show median and interquartile range.

Abbreviations: Gal-I, galectin-I; ISS, international staging system; NS, not significant.

at diagnosis and at 3 months after HDT or for the change in Gal-1 during the course of treatment (all $P>0.05$ ).

To investigate whether serum Gal-1 was a prognostic marker in the cohort of patients with MM, we performed a Cox proportional hazards regression analyses with Gal-1 as a continuous variable, showing that Gal-1 was not a prognostic factor, for patients treated neither with MP $(n=61$, hazard ratio $[\mathrm{HR}]=0.99, P=0.97)$ nor with $\mathrm{HDT}(\mathrm{n}=41$, $\mathrm{HR}=1.02, P=0.90)$.

\section{Gal-I and bone marrow angiogenesis}

Previously, we have shown that high levels of bone marrow angiogenesis are associated with poor outcome in MM. ${ }^{18}$ In Figure 3, serum Gal-1 levels are plotted by angiogenesis level, using our previously published data on bone marrow angiogenesis measured as MVD (split on median). There was a trend toward slightly higher serum Gal-1 levels in patients with highest MVD, but the difference was not statistically significant $(P=0.11)$.

In our previous study, serum levels of syndecan-1 associated positively with bone marrow angiogenesis and with

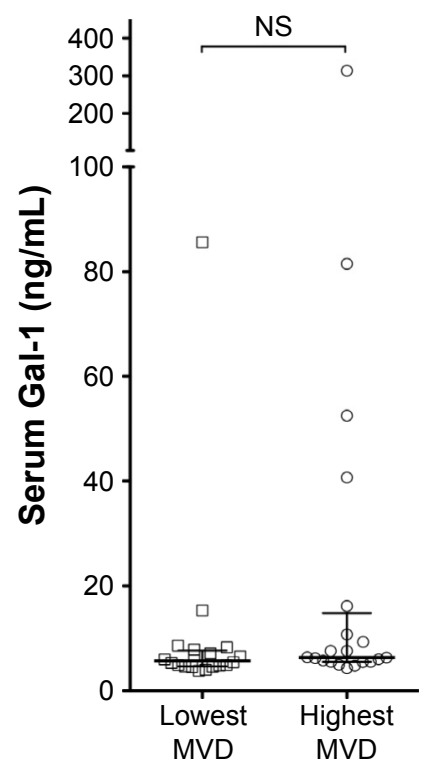

Figure 3 Serum Gal-I and bone marrow angiogenesis.

Notes: Serum Gal-I levels at time of diagnosis, stratified by bone marrow angiogenesis level measured as MVD, spilt on median MVD. Bars show median and interquartile range.

Abbreviations: Gal-I, galectin-I; MVD, micro-vessel density; NS, not significant.

poor patient outcome. ${ }^{18}$ In this study, we also found a positive linear association of serum Gal-1 with syndecan-1 levels (Figure 4A, $\mathrm{n}=49, R=0.36, P=0.01$ ).

\section{Gal-I and markers of macrophage activation}

Because Gal-1 may be produced by myeloid cells, as reported for patients with CLL, ${ }^{11}$ and may induce M2 polarization of macrophages, we examined possible associations with monocyte/macrophage-derived sCD163 and sMR. At the time of diagnosis, serum Gal-1 was positively associated with SCD163 (n=102, $R=0.24, P=0.02$ [Figure 4B]). However, there was no association of Gal-1 with $\operatorname{sMR}(n=102, P=0.92)$. At 3 months after HDT, there was a borderline significant association with both $\mathrm{sCD} 163(P=0.07)$ and $\operatorname{sMR}(P=0.05$; data not shown).

\section{Discussion}

Recent years have seen a surge in the interest of galectin involvement in cancer pathophysiology, ${ }^{2}$ and blood levels of Gal-1 have been associated with more severe disease in hematological malignancies..$^{9,11}$

However, serum levels of Gal-1 have not previously been reported in patients with MM. In a cohort of 102 patients with newly diagnosed, treatment demanding MM, we found no association between serum Gal-1 at diagnosis, tumor burden (bone marrow PC infiltration), or with overall patient survival. These findings indicate that Gal-1 is not an 

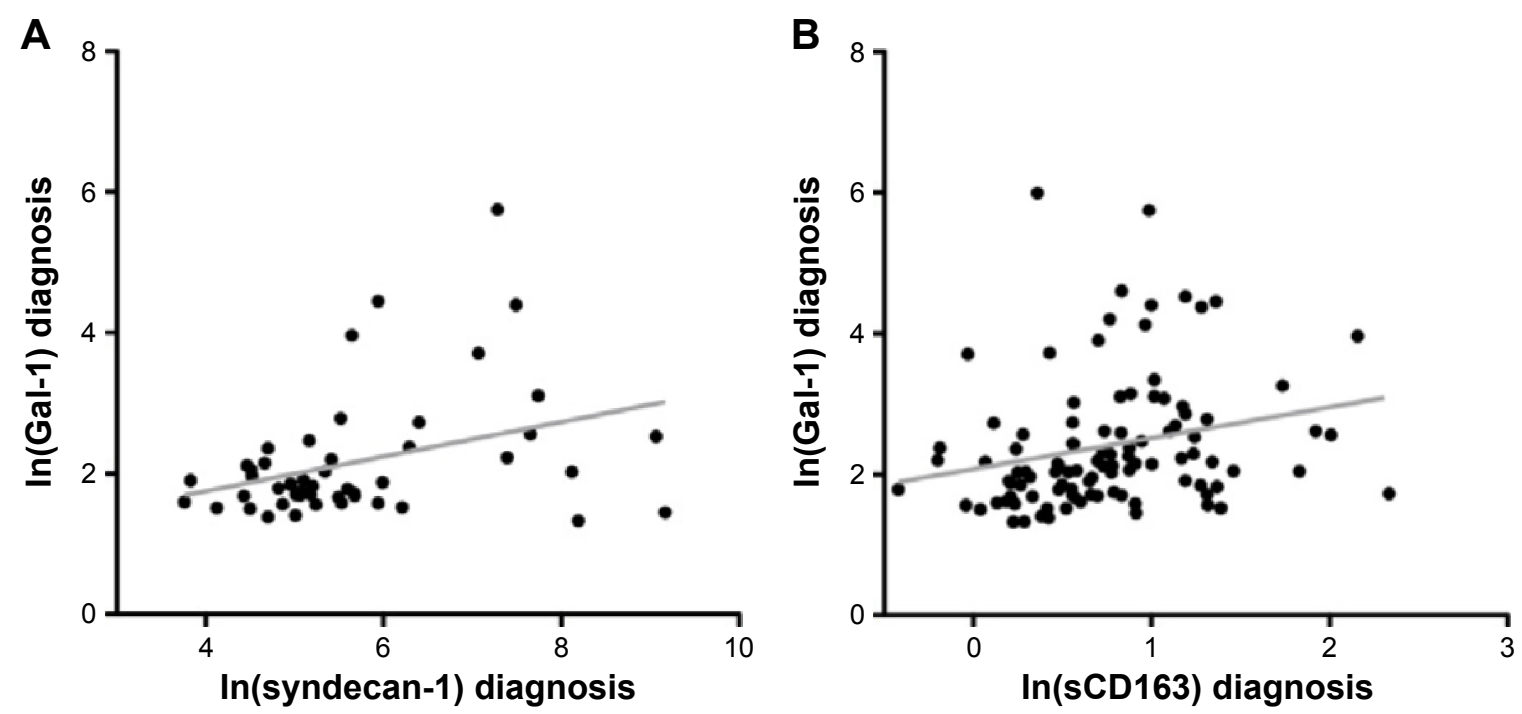

Figure 4 Associations of serum Gal-I with macrophage and plasma cell markers.

Notes: Serum Gal-I levels at time of diagnosis in patients with multiple myeloma were positively associated with levels of $(\mathbf{A})$ serum syndecan- $I$ (sCDI38, $R=0.36, P=0.0 \mathrm{I})$ and (B) serum sCDI63 $(R=0.24, P=0.02)$. Plotted parameters were log-transformed using the natural logarithmic function.

Abbreviations: Gal-I, galectin-I; sCDI38, soluble CDI38.

important serum biomarker in MM, in contrary to what has been shown for cHL and CLL. ${ }^{9,11}$ This is further supported by the finding that serum Gal-1 levels were not increased in MM patients compared with healthy controls, opposed to the results in cHL and CLL.

In a recent study, high expression of Gal-1 mRNA expression in purified $\mathrm{CD} 138^{+}$bone marrow cells correlated with worse outcome for MM patients. ${ }^{17}$ Gal-1 is expressed by endothelial cells and has been shown to be a proangiogenic growth factor, ${ }^{19}$ and in this study, there was a trend toward higher Gal-1 in patients with high MVD, but there was no statistically significant association between the two parameters. Storti et al reported high Gal-1 expression in hypoxic conditions, and inhibition of Gal-1 in MM cells decreased expression of proangiogenic genes and reduced tumor burden and angiogenesis (MVD) in mouse models. ${ }^{17}$ Thus, this study indicates an important role for Gal- 1 in $\mathrm{CD} 138^{+}$cells, but from our results, this may not be translated into measurable Gal-1 protein in serum. Hence, the limitation of this study is that we only had access to blood samples, and not samples of bone marrow. Therefore, future studies on Gal-1 in MM should include bone marrow samples for gene expression analysis, and, especially, protein level analyses on CD138 ${ }^{+}$ cells may provide further insight into the role of Gal-1 in MM pathophysiology.

In CLL and cHL, Gal-1 is expressed by both malignant and tumor-supporting cells. ${ }^{8,11}$ Gal-1 may influence the activation state of macrophages inducing a less inflammatory (M2-like) phenotype, which has been associated with poor outcome in several human cancers. ${ }^{23}$ In cHL, tissue expression of Gal-1 was correlated with CD163, which is a marker of M2-activated macrophages. ${ }^{8}$ In MM, higher infiltration of CD163-expressing macrophages is associated with shorter survival, ${ }^{14}$ and we have previously shown that sCD163 is associated with overall survival in MM. ${ }^{20}$ In this study, we found a positive association between the levels of serum Gal-1 and sCD163, which support the previous findings indicating that Gal-1 may be associated with alternative macrophage activation. ${ }^{5}$

Thus, one explanation for the present results may be that the increased mRNA expression of Gal-1 in MM CD138+ bone marrow cells ${ }^{17}$ is not translated into increased protein levels, or that the increase is not reflected in the peripheral blood, resulting in serum levels not being significantly higher in patients with MM than in healthy controls. This should be investigated in future studies.

In conclusion, the serum levels of Gal-1 were not increased in patients with MM (compared with healthy donors) and did not show association with known prognostic factors or patient survival, which is in contrast to published data from patients with other hematological malignancies. However, serum Gal-1 levels were associated with monocyte/ macrophage-derived sCD163, supporting the involvement of Gal-1 in alternative (M2-like) activation of macrophages.

\section{Author contributions}

MNA contributed in conception and design, collection of data, analysis and interpretation of data, drafting the article, and approval of the version to be published. ML contributed to conception and design, collection of data, revising 
the manuscript critically, and approval of the version to be published. NA contributed in collection of data, revising the manuscript critically, and approval of the version to be published. IP carried out providing Gal-1 data on healthy donors, revising the manuscript critically, and approval of the version to be published. RH performed Gal-1 assay development and validation, revising the manuscript critically, and approval of the version to be published. MB performed Gal-1 assay development and validation, revising the manuscript critically, and approval of the version to be published. BH contributed in Gal-1 assay development and validation, revising the manuscript critically, and approval of the version to be published. HJM carried out collection of data, revising the manuscript critically, and approval of the version to be published. NFA contributed to conception and design, collection of data, analysis and interpretation of data, revising the manuscript critically, and approval of the version to be published.

\section{Disclosure}

The authors report no conflicts of interest in this work.

\section{References}

1. Liu F-T, Rabinovich GA. Galectins: regulators of acute and chronic inflammation. Ann N Y Acad Sci. 2010;1183:158-182.

2. Giordano M, Croci DO, Rabinovich GA. Galectins in hematological malignancies. Curr Opin Hematol. 2013;20(4):327-335.

3. Rabinovich GA, Vidal M. Galectins and microenvironmental niches during hematopoiesis. Curr Opin Hematol. 2011;18(6):443-451.

4. Barrionuevo P, Beigier-Bompadre M, Ilarregui JM, et al. A novel function for galectin-1 at the crossroad of innate and adaptive immunity: galectin-1 regulates monocyte/macrophage physiology through a nonapoptotic ERK-dependent pathway. J Immunol. 2006;178(1): 436-445.

5. Yang RY, Rabinovich GA, Liu FT. Galectins: structure, function and therapeutic potential. Expert Rev Mol Med. 2008;10:e17.

6. Rubinstein N, Alvarez M, Zwirner NW, et al. Targeted inhibition of galectin-1 gene expression in tumor cells results in heightened $\mathrm{T}$ cellmediated rejection. Cancer Cell. 2004;5(3):241-251.

7. Toscano MA, Bianco GA, Ilarregui JM, et al. Differential glycosylation of TH1, TH2 and TH-17 effector cells selectively regulates susceptibility to cell death. Nat Immunol. 2007;8(8):825-834.
8. Kamper P, Ludvigsen M, Bendix K, et al. Proteomic analysis identifies galectin-1 as a predictive biomarker for relapsed/refractory disease in classical Hodgkin lymphoma. Blood. 2011;117(24):6638-6649.

9. Ouyang J, Plütschow A, Pogge von Strandmann E, et al. Galectin-1 serum levels reflect tumor burden and adverse clinical features in classical Hodgkin lymphoma. Blood. 2013;121(17):3431-3433.

10. Petruskevicius I, Ludvigsen M, Hjortebjerg R, et al. Clinical relevance of galectin-1 in hematologic malignancies treated with non-myeloablative hemopoietic stem cell transplantation. Bone Marrow Transplant. 2016; 51(10):1387-1390.

11. Croci DO, Morande PE, Dergan-Dylon S, et al. Nurse-like cells control the activity of chronic lymphocytic leukemia B cells via galectin-1. Leukemia. 2013;27(6):1413-1416.

12. Kyle RA, Steensma DP. History of multiple myeloma. Recent Results Cancer Res. 2011;183:3-23.

13. Zheng Y, Yang J, Qian J, et al. PSGL-1/selectin and ICAM-1/CD18 interactions are involved in macrophage-induced drug resistance in myeloma. Leukemia. 2012;27(3):702-710.

14. Suyanı E, Sucak GT, Akyürek N, et al. Tumor-associated macrophages as a prognostic parameter in multiple myeloma. Ann Hematol. 2013;92(5): 669-677.

15. Panchabhai S, Kelemen K, Ahmann G, Sebastian S, Mantei J, Fonseca R. Tumor-associated macrophages and extracellular matrix metalloproteinase inducer in prognosis of multiple myeloma. Leukemia. 2016; 30(4):951-954.

16. Abroun S, Otsuyama K-I, Shamsasenjan K, et al. Galectin-1 supports the survival of CD45RA(-) primary myeloma cells in vitro. Br J Haematol. 2008;142(5):754-765.

17. Storti P, Marchica V, Airoldi I, et al. Galectin-1 suppression delineates a new strategy to inhibit myeloma-induced angiogenesis and tumoral growth in vivo. Leukemia. 2016;30(12):2351-2363.

18. Andersen NF, Standal T, Nielsen JL, et al. Syndecan-1 and angiogenic cytokines in multiple myeloma: correlation with bone marrow angiogenesis and survival. Br J Haematol. 2005;128(2):210-217.

19. Griffioen AW, Thijssen VL. Galectins in tumor angiogenesis. Ann Transl Med. 2014;2(9):90.

20. Andersen MN, Abildgaard N, Maniecki MB, Møller HJ, Andersen NF. Monocyte/macrophage-derived soluble CD163: a novel biomarker in multiple myeloma. Eur J Haematol. 2014;93(1):41-47.

21. Andersen MN, Andersen NF, Rødgaard-Hansen S, Hokland M, Abildgaard N, Møller HJ. The novel biomarker of alternative macrophage activation, soluble mannose receptor (sMR/sCD206): implications in multiple myeloma. Leuk Res. 2015;39(9):971-975.

22. Greipp PR, San Miguel J, Durie BGM, et al. International staging system for multiple myeloma. J Clin Oncol. 2005;23(15):3412-3420.

23. Sica A, Allavena P, Mantovani A. Cancer related inflammation: the macrophage connection. Cancer Lett. 2008;267(2):204-215.
OncoTargets and Therapy

\section{Publish your work in this journal}

OncoTargets and Therapy is an international, peer-reviewed, open access journal focusing on the pathological basis of all cancers, potential targets for therapy and treatment protocols employed to improve the management of cancer patients. The journal also focuses on the impact of management programs and new therapeutic agents and protocols on

Submit your manuscript here: http://www.dovepress.com/oncotargets-and-therapy-journal

\section{Dovepress}

patient perspectives such as quality of life, adherence and satisfaction. The manuscript management system is completely online and includes a very quick and fair peer-review system, which is all easy to use. Visit http://www.dovepress.com/testimonials.php to read real quotes from published authors. 